\title{
The Primary Goal of Anticoagulation in Atrial Fibrillation to Prevent Stroke and Not to Offer the Apparently Cheapest Treatment
}

\author{
Hans-Christoph Diener \\ Department of Neurology and Stroke Center, University Hospital Essen, Germany
}

Patients with atrial fibrillation $(\mathrm{AF})$ have a particular high risk of cardio-embolic stroke. Cardio-embolic strokes have a high mortality $(20 \%)$ and $>50 \%$ of patients remain permanently disabled. Oral anticoagulation with warfarin is highly effective in preventing strokes as long as warfarin is taken. Unfortunately many patients with atrial fibrillation refuse to take warfarin because they are afraid of major bleeding complications in particular intracranial bleeds. In addition long term compliance and adherence with warfarin is terrible due to the need of regular international normalized ratio (INR) monitoring and the many interactions with food and drugs in elderly patients with polypharmacy. Anticoagulation is most effective in old patients who have difficulties to get to a physicians' office or an anticoagulation clinic to get the INR monitored. Just imagine the 86 years old lady with unsteady gate and a walker needing to get to a doctor in winter with ice and freezing temperatures outside.

Data from Canadian and German stroke units on admission of patients with $\mathrm{AF}$ and a new stroke display shocking facts. ${ }^{1,2}$ One third of patients with AF and without contraindications for anticoagulation are untreated at the time of stroke. Thirty percent are treated with aspirin which is not effective in secondary stroke prevention in $\mathrm{AF}^{3}$ Another $30 \%$ are treated with warfarin but the INR was $<2.0$ at the time of stroke. Only $10 \%$ of patients were on warfarin and the INR was between 2.0 and 3.0.

Reimbursement bodies, however, will consider only the last $10 \%$ of patients, namely patients with AF well controlled on warfarin. This does not reflect clinical reality. The most restrictions for the use of novel anticoagulants (NOACs) instead of warfarin prevent the patients in highest need of stroke preven- tion from a drug that is superior to warfarin and has fewer intracranial bleeds, namely the elderly.

In this issue, Bang et al. ${ }^{4}$ summarized pieces of evidence that NOACs may be particularly helpful for Asian stroke patients. Indeed, Asians are at particular high risk of major bleeds on warfarin. This risk is much lower with novel anticoagulants. Therefore it is not rational to restrict the use of NOACs in Asian patients with AF. My policy would be to offer all patients who had a transient ischemic attack or ischemic stroke and AF a NOAC, because these patients have a particular high risk of a recurrent stroke. In primary prevention I would offer NOACs to untreated patients, patients on aspirin and patients poorly controlled on warfarin. I see no reason to switch well controlled patients on warfarin to NOACs. In summary putting high hurdles on the prescription of NOACs will lead to many strokes with severe disability and tremendous costs for a national health care system.

\section{References}

1. Gladstone DJ, Bui E, Fang J, Laupacis A, Lindsay MP, Tu JV, et al. Potentially preventable strokes in high-risk patients with atrial fibrillation who are not adequately anticoagulated. Stroke 2009;40:235-240.

2. Weimar C, Benemann J, Katsarava Z, Weber R, Diener HC. Adherence and quality of oral anticoagulation in cerebrovascular disease patients with atrial fibrillation. Eur Neurol 2008;60: 142-148.

3. European Atrial Fibrillation Trial (EAFT) Study Group. Secon- 
dary prevention in non-rheumatic atrial fibrillation after transient ischaemic attack or minor stroke. Lancet 1993;342:12551262.

4. Bang OY, Hong KS, Heo JH, Koo J, Kwon SU, Yu KH, et al. New oral anticoagulants may be particularly useful for Asian stroke patients. J Stroke 2014;16:73-80.
Correspondence: Hans-Christoph Diener Department of Neurology and Stroke Center, University Hospital Essen, Hufeland Strasse 55, Essen 45122, Germany

Tel: +492017232460, Fax: +492017235901

E-mail: hans.diener@uk-essen.de 